\title{
Análisis teórico del proceso de intervención psicológica en el deporte
}

\section{Theoretical analysis of the process of psychological intervention in the sport}

\section{Analises teórica do processo de intervenção psicológica no esporte}

\author{
Eugenio A. Pérez-Córdoba
}

Universidad de Sevilla, España

\begin{abstract}
Resumen: En la Universidad aprendemos las técnicas más usadas para la intervención en psicológica, en nuestro caso en el deporte y el ejercicio. También aprendemos las bases teóricas en las que se asientan dichas técnicas. Incluso aprendemos a realizar estadísticas adecuadas y desarrollar procedimientos metodológicos adecuados de intervención, pero rara vez analizamos el proceso de la propia intervención en sí mismo. En este trabajo realizaremos ese análisis basándonos en las competencias profesionales que debe tener un psicólogo según nos plantea la EFPA (Federación Europea de Asociaciones de Psicología), de manera que el lector pueda entender que, para poder intervenir, adecuadamente claro, debe poseer no solo esos conocimientos, sino, también, unas competencias adecuadas para poder llevar a la práctica los conocimientos teóricos obtenidos durante su periodo estudiantil. Este análisis empezará en la primera toma de contacto y terminará con la presentación del informe final, pasando por la evaluación y el diseńo y puesta en práctica de la intervención diseñada.

Palabras clave: Competencias profesionales, psicología del deporte, intervención.

Abstract: In college we learn the techniques used for psychological intervention, in our case in sport and exercise. Also learn the theoretical basis on which these techniques are based. Even learn to perform appropriate statistical procedures and develop appropriate methodological intervention, but rarely analyze the process of the intervention itself in itself. In this research work that analysis based on the skills that you need a psychologist as
\end{abstract}

we face the EFPA (European Federation of Psychology), so that the reader can understand that in order to intervene appropriately clear, must possess not only such knowledge, but also adequate competences to implement the knowledge obtained during their student. This analysis will begin in the first contact and ends with the final report, through the evaluation and the design and implementation of intervention designed.

Key words: Professional skills, sport psychology, intervention.

Resumo: Na Universidade aprendemos as técnicas mais utilizadas para a intervenção psicológica no âmbito do esporte e do exercício físico. Também aprendemos as bases teóricas das fichas técnicas, incluso aprendemos a realizar estatísticas adequadas e desenvolver procedimentos metodológicos adequados de intervenção, mas dificilmente analisamos o processo da própria intervençấo. Nesse trabalho realizaremos uma analises baseando nas competências profissionais que deve ter um psicólogo de acordo com a EFPA (Federaçáo Europeia de Associaçóes de Psicologia), dessa maneira o leitor entenderá que para poder intervir adequadamente deverá possuir umas capacidades para realizar na pratica os conhecimentos teóricos adquiridos durante sua formação universitária. Esta analise começará com o primeiro contato e terminará com a apresentação de um informe final, passando pela avaliaçáo, o desenho e a posta em pratica da intervençáo desenhada.

Palavras Chave: Capacidades profissionais, psicologia do esporte, intervenção.
En este trabajo vamos a revisar el proceso de intervención del psicólogo del deporte. Para ello nos basaremos en las competencias profesionales que propone la Federación Europea de Asociaciones de Psicólogos Profesionales (EFPPA) la cual plantea una serie de competencias, que debe poseer el profesional que se encarga de aplicar los conocimientos psicológicos (Infocop online, 2008), en nuestro caso, al deporte y el ejercicio.

La competencia debe definirse, según Roe (2002) como "una capacidad aprendida para realizar adecuadamente una tarea, función o rol”, es decir, que las competencias definen las capacidades que debe poseer el futuro profesional para poder ser efectivo en su labor aplicando la psicología en las diferentes áreas de intervención de la psicología de la actividad física y el deporte.

Dirección para correspondencia: Departamento de Psicología Experimental, Universidad de Sevilla.

Calle Camino José Cela s/n .41018. e-mail: ecordoba@us.es
En nuestro análisis revisaremos esas competencias o capacidades analizando el proceso de intervención psicológica desde el primer contacto con el cliente hasta la realización del informe final, el lector interesado puede leer también, para el tema de las competencias, Pérez Córdoba (2010) y Pérez Córdoba (2012a).

En esta revisión, además de definir y describir las tareas propias del profesional, las compararemos con las que realiza otro profesional de la salud como es el médico, o con un profesional del aprendizaje como es un profesor, no sin dejar de exponer, a modo de ejemplo, el posible proceso de intervención psicológica en un supuesto con un deportista.

\section{Toma de contacto y definición operativa del problema}

Lo primero que ocurre en el proceso de intervención -en cualquier proceso de relación se podría decir- es la toma de contac- 
to. Durante esta primera visita, se deben identificar las variables relevantes, en base a los síntomas descritos por el cliente.

Es evidente que, en un primer momento, el sujeto que nos visita puede no saber describirnos exactamente el problema usando una terminología científica y correcta. Más bien suele ocurrir que nos explica, a su manera, los síntomas y los comportamientos que él considera como "problema" a solucionar, estableciendo falsas atribuciones y generando expectativas propias que, a menudo, no coinciden con la realidad.

Con frecuencia será necesaria una reformulación de las demandas -y de las expectativas- del usuario, de manera que se pueda operativizar el problema en cuestión y puedan especificarse, de manera realista, las posibles metas a conseguir tras la intervención.

En nuestro trabajo iremos estableciendo comparaciones con el trabajo de un profesional de la salud, como es el médico, y con un profesional de la educación, como sería un profesor, aunque queremos dejar claro en este punto que nuestro enfoque en psicología del deporte ha ido cambiando desde un enfoque más terapéutico de intervención hacia un enfoque más educativo y de aprendizaje, basado en el entrenamiento de habilidades psicológicas.

Así, y en un símil con un médico debemos reconocer nuestra propia ignorancia al exponer al galeno nuestras dolencias. De esta forma, suele ser normal que, de manera genérica, hablemos con el profesional y le digamos que tenemos un "resfriado" -en un lenguaje mucho más coloquial que "tenemos un trancazo de padre y muy señor mio"-, cuando, en realidad al médico no le damos una información precisa de nuestra dolencia, sino que usamos un lenguaje coloquial que hace mención, de manera genérica a unos ciertos síntomas, dado que el término "resfriado" es un término coloquial y no científico.

No es el momento de realizar un análisis detallado sobre si nuestro lenguaje psicológico es científico o coloquial (Pérez Córdoba y Caracuel, 1995), pero baste ahora con señalar que términos como motivación, atención o emoción son palabras que no están definidas por la psicología, de una forma unívoca $-y$ por tanto científica- siendo muchas las referencias a las que puede hacer mención cada uno de esos conceptos.

Siguiendo con el caso del profesional médico, y dado que en esta fase del proceso es en la que debemos obtener el máximo de información de cara al planteamiento de acciones concretas de intervención, el médico debe informarse mediante una anamnesis y auscultarnos para comprobar si "nuestro resfriado" es de las vías altas o si tenemos algún problema centrado en los pulmones, o, simplemente, es una pequeña obstrucción pasajera de las vías respiratorias. Es decir, debe diagnosticar, con más exactitud, nuestro problema, para poder realizar una definición operativa de la dolencia que le permita, posteriormente, realizar una adecuada intervención.

Ese paso de obtención de una mayor información ya podría entrar, más exactamente, en el siguiente paso -eva- luación y psicodiagnóstico-, aunque en esta primera fase de análisis de las necesidades del cliente, se deben clarificar las demandas hasta un punto en el que se puedan determinar las acciones significativas a realizar.

En el símil médico que estamos utilizando, conviene señalar que, dada la escasez de tiempo del que disponen en sus consultas, toma de contacto y evaluación suelen coincidir en nuestra primera visita, incluso podríamos decir que, en esta primera -y a veces única- visita ya se produce el diagnóstico y la prescripción de la intervención. Eso sí, siempre que el problema no revista la suficiente gravedad como para que se haga necesario una evaluación y diagnóstico mas específico -pruebas analíticas, radiografías, etc.

Si habláramos del profesional de la educación, en la primera entrevista con los tutores para determinar el objetivo de la intervención, suele ser normal que los padres no sepan definir, con exactitud, el problema especifico, sino que, más bien, suelen plantear problemas generales de adaptación a la escuela o de rendimiento en alguna materia en concreto -o en general-, pues, hemos de entender, que ellos no son profesionales, sino que somos nosotros los verdaderos profesionales competentes para definir el verdadero objetivo de nuestra intervención.

Es decir, que, si queremos realizar una actuación eficaz, ya sea el profesional que fuese, no debemos olvidar que, lo primero, es delimitar adecuadamente el objetivo de nuestra intervención, debiendo ser descrito el tratamiento solo después de este paso, y no antes, como por desgracia, hacemos con una mala praxis, en la que damos soluciones antes de haber definido operativamente el problema (y es entonces cuando nos comparan con unos simples "amigos" del cliente, no sin razón, pues los amigos, casi sin escucharnos, ya nos dicen lo que ellos harían....)

Diferenciando en nuestro caso ambos pasos -toma de contacto y evaluación-, aunque puedan, a veces, darse en el mismo día, es, en esta primera toma de contacto, el momento de realizar una adecuada anamnesis, recabando el máximo de información del cliente $-\mathrm{y}$ si es posible de otras personas que le rodean-, pues, como veremos en el siguiente apartado, no solo hemos de evaluar al sujeto -deportista en nuestro caso- sino también a las circunstancias.

Esto nos permitirá construir un buen modelo explicativo de las relaciones que se producen en el ámbito deportivo, y es que, no debemos olvidar, como nos comenta Riera (1985) que lo que debe estudiar la psicología del deporte son las relaciones que se producen en el ámbito del deporte y el ejercicio.

Continuando con nuestro análisis de esta primera toma de contacto, no debemos olvidar que, además de analizar la demanda y de especificar el objetivo de nuestra intervención, hemos de establecer una adecuada empatía con el cliente, pues si no se establece esta adecuada confianza entre ambos, difícilmente podremos conseguir nuestros objetivos. A 
este respecto habría que añadir que esta buena relación y la consecución de la adecuada y necesaria confianza puede que necesite más tiempo que el que se utiliza en una primera -o única- sesión de toma de contacto.

También debemos indicar que, al igual que nosotros observamos y evaluamos, estamos siendo observados y evaluados por el cliente, y de esta evaluación puede depender el resto del proceso -si es que se deciden a seguir con nosotros.

En este sentido, también es importante determinar si el propio profesional está capacitado para aportar valor y responder de forma adecuada para satisfacer esa demanda. Así, en esta fase de analizar necesidades y establecer objetivos, hemos de analizar, además, los recursos necesarios y disponibles para incrementar la probabilidad de éxito y las garantías de calidad de la intervención, incluyendo nuestras propias competencias y habilidades.
No queremos terminar este apartado sin indicar las diferencias entre trabajar en una consulta individual, con un proceso de intervención mas parecido a lo que estamos describiendo aquí, y trabajar en una organización o club, en el que debemos de asegurarnos de que, nuestra intervención, no supone un conflicto con la organización que nos contrata, siendo necesario, si es este el caso, analizar también las necesidades de la organización y las relaciones de poder formales e informales, las comunicaciones, y, la distribución de recursos asociadas a éstas, en general, que se producen en este tipo de complejas organizaciones. Y, por ende, deberemos ajustar los objetivos a conseguir con los individuos y los de la organización de manera que encajen entre ambos y no supongan un conflicto mas a añadir a los que ocasionaron la consulta.

Cuadro 1. Toma de contacto y definición operativa del problema en un deportista.

\section{Ejemplo}

En estos pequeños cuadros iremos analizando un supuesto de un deportista que llega a nuestra consulta para que le intentemos ayudar con su problema.

En esta ocasión expondremos el caso de un futbolista, delantero centro mas específicamente, que marcaba muchos goles de saque de esquina, pero que últimamente no acierta ni una.

Es un caso que nos llega por la información que nos proporciona su entrenador, quien añade que cree que su futbolista esta "ansioso". Pese a que le preguntamos, toda la información que nos aporta redunda en el hecho de que, últimamente, lo encuentra mas "nervioso" de la cuenta.

Dado que necesitamos mas información, para poder establecer una adecuada definición operativa del problema, acudimos a la prensa escrita en la que encontramos continuas alusiones a la "falta de concentración" de ese deportista.

No contentos con dicha información -aportada por entrenador y prensa- acudimos a un antiguo profesor nuestro, quien nos impartía una asignatura en la que se analizaban los pensamientos. Dicho profesor nos asegura que, muy probablemente, sea un problema de pensamientos negativos.

Una última información recabamos sobre el posible problema, y acudimos a un compañero de promoción quien nos afirma que, en su opinión, podría ser un problema de "motivación extrínseca frente a la intrínseca".

Ante tan diferente información - ansiedad, concentración, pensamientos negativos y motivación- no sabemos, con exactitud, cual es el problema, definido de una forma operativa, por lo que decidimos obtener más información pero de una forma más precisa, y preguntamos a un entrenador experto - nosotros somos psicólogos, no expertos en ese deporte- sobre cuál es la mejor forma de conseguir un gol tras un saque de esquina. Este nos dice que lo mejor es "estar atento al que saca el balón, a la portería y el portero y a los posibles contarios que estén cerca del que intenta rematar".

Con esta información ya podemos, al menos, establecer el objetivo último de nuestra intervención que es, ni más ni menos, conseguir que el comportamiento de dicho jugador se parezca lo más posible al descrito por el experto como "ideal".

En este punto conviene recalcar que no somos expertos en tal o cual deporte, pero sí expertos en "cambiar comportamientos", de manera que, si evaluamos -en la fase siguiente- cuál es el comportamiento actual del jugador, y lo comparamos con el "óptimo" podremos definir, con más exactitud, los pasos necesarios para ese cambio de comportamiento, y podremos diseńar intervenciones concretas y específicas, para promover ese cambio de comportamiento.

\section{Evaluación y psicodiagnóstico}

Una segunda fase del proceso -insistimos en que las fases no tienen por qué coincidir con las sesiones- sería la de evaluación y psicodiagnóstico, entendiendo que no solo hemos de evaluar a los individuos, sino que, además, hemos de diagnosticar al grupo y a la organización a la que pertenecen, y el contexto, en nuestro caso deportivo, en el que desarrollan su comportamiento. 
Así, si bien antes hemos realizado una primera toma de contacto y una primera estimación de las necesidades del cliente para poder operativizar el problema, es ahora cuando se realiza una mas detallada evaluación y diagnóstico de dicho problema.

De esta forma, y en el símil médico, si este ha decidido que, pese a su auscultación y anamnesis, sería conveniente realizar unas analíticas para evaluar con más detalle el problema, es, en esta fase, en la que se solicita dicha evaluación.

Hemos de ańadir que, para esta evaluación, el personal médico cuenta con otros profesionales -diplomados en enfermería- que realizan algunas de estas labores, tales como la extracción de sangre y el análisis bioquímico de ésta, mientras que, en el caso del psicólogo, no solemos disponer de estos ayudantes, si bien es cierto que, por ejemplo, para una observación y registro del comportamiento, podemos contar - con suerte- con ayudantes que nos registren dichas conductas.

En el caso que nos ocupa, la labor del psicólogo del deporte, disponemos de cuestionares, tests, procedimientos de observación, etc., adecuados para la realización de esta más completa evaluación.

Hemos de añadir que, esta evaluación de comportamientos, debe realizarse, preferentemente, en el contexto en el que nos ocupa, en nuestro caso, el deportivo, pues sabido es que los comportamientos son relaciones entre el sujeto y el contexto y que pueden darse comportamientos diferentes del mismo sujeto en diferentes contextos, siendo el objetivo de nuestro trabajo evaluar los comportamientos, las relaciones, en los contextos lo mas similares a los reales, siendo lo ideal que se observen en dichas conductas en los contextos en que se han de producir (Pérez Córdoba, 2011)

Siguiendo con el símil médico, aunque con sus diferencias obvias, no olvidemos que los médicos han de preguntar, al menos, por el contexto del paciente, pues puede que su "resfriado" se debe a carencias en dicho contexto o a simples contagios de otros miembros de la familia, debiéndose analizar, en algunos casos, incluso los antecedentes familiares, pues pueden transmitirse enfermedades de padres a hijos.

En el caso de la psicología, y sin llegar a esos extremos, sí que podemos convenir en que el contexto en el que se desarrolla el comportamiento es tan importante -ni mas ni menos- que el propio organismo o persona que analizamos, y que conviene evaluar no solo el contexto -elementos físicos, otras personas, normas, etc.-, sino, que, además, resulta importante evaluar al grupo y sus relaciones -el equipo deportivo- y a la organización en la que se desarrollo el comportamiento -club- pues, como venimos apuntando en todo este trabajo, hay que analizar las relaciones que se producen en el ámbito deportivo, complejo sistema, compuesto, a su vez, por múltiples subsistema.

En este sentido, la evaluación del contexto, nos acercamos más a la labor del profesional de la educación que al de la medicina, aunque debemos convenir que, en ambos tipos de profesionales -tres si contamos el de la psicología del deporte-, la evaluación debe realizarse tanto de la persona como del contexto, pues lo que nos interesa a los psicólogos, como estamos repitiendo una y otra vez, es la relación.

Cuadro 2. Evaluación y psicodiagnóstico.

Siguiendo con el ejemplo del futbolista que no marca goles, diseñaríamos una hoja de observación y registro de dicho comportamiento, entendiendo que hay comportamientos que no podemos observar (pensamientos, comportamiento verbal, autoinstrucciones, etc.) y que debemos cuestionar a nuestro deportista para poder obtener dicha información.

También se le podrían pasar cuestionarios y tests específicos para valorar su nivel de atención y de ansiedad, así como preguntas específicas para conocer su tipo de motivación, etc., entendiendo que hay que tener información sobre lo que hace (observación) y sobre lo que dice (cuestionarios, test, etc.) entendiendo que ambas informaciones son parciales.

En esta fase de evaluación no debemos olvidar la evaluación de los aspectos del grupo y de la organización, tales como relaciones de grupo, posición en la tabla del equipo, objetivos del club, etc., pues podrían ayudarnos a definir mejor nuestra intervención.

En esta evaluación intentamos analizar su comportamiento y lo comparamos con el óptimo, detectando que dicho futbolista "piensa" justo cuando van a lanzar el saque de esquina, y que lo que piensa es que si marca el gol puede conseguir ser pichichi, centrando, por tanto, su atención en el tiempo que falta para acabar el partido y en la posible prima que le daría el club por conseguir la victoria.

Podemos comprobar, también, que este comportamiento no se parece en nada al "ideal" definido por el técnico o experto en dicho comportamiento, pues se dan comportamientos que "sobran" (pensar en pichichi, etc.) y "faltan" comportamientos que instaurar (autoinstrucciones para centrarse en tarea, en balón, portero, etc.) 
Esto sería similar a la analítica en la que el médico detecta que "sobra” azúcar y que "falta” hierro. Evaluación que le permitirá al médico implementar las medidas oportunas para corregir dichas "diferencias" con el "estado ideal" -fisiológicamente hablando- del cliente. Como es obvio, gracias a nuestra analítica, sabemos lo que falta y sobra-psicológicamente hablando- y podremos, en la siguiente fase, implementar las medidas adecuadas para que el comportamiento deportivo se asemeje lo mas posible al "ideal" (según ese técnico, claro).

Como conclusión o diagnóstico, objetivo de esta fase, no debemos olvidar que podemos establecer diferentes niveles de diagnóstico, el individual, el colectivo, el organizacional, pues, de cara al diseńo del trabajo en la fase siguiente, debemos tener claro que todo puede influir sobre todo (mulifactorial e interdependiente).

\section{Definición de la intervención}

Una vez que hemos definido operativamente el problema, y hemos hecho todas las evaluaciones pertinentes, debemos proceder a desarrollar el producto o servicio psicológico con el que vamos a intervenir, aunque no debemos olvidar que la evaluación, por sí misma, ya es una intervención (pues es una variable que "interviene" en el proceso...)

Para ello son necesarios todos nuestros conocimientos básicos sobre psicología, pues hemos de diseñar nuestra intervención a partir de la teoría y de los métodos psicológicos, y no de forma intuitiva y poco sistemática.

No olvidemos que, si queremos ser profesionales, y por tanto diferentes a los mal llamados "psicólogos de calle" no podemos, ni debemos, basar nuestras intervenciones en la casualidad ni en la "suerte" de que los factores que vamos a trabajar "coincidan" adecuadamente, sino que hemos de disponer dichos factores de una forma sistemática y coherente para que se produzcan los cambios esperados.

Continuando con el símil médico ¿̨acaso el médico receta los componentes químicos, denominados pastillas, de una forma aleatoria?

Es obvio que no, más bien las prescribe basándose en los conocimientos ampliamente desarrollados y experimentados por la ciencia médica, de manera que, una vez evaluado lo que falta o lo que sobra, simplemente nos receta que tomemos aquellos componentes que nos faltan -v.g. hierro- y que dejemos de tomar aquellos otros elementos que nos sobran -por ejemplo, azúcar. A veces nos prescribe otros elementos que potencian la desaparición de los unos o fomentan la asimilación de los otros.

En nuestra ciencia no prescribimos productos químicos, pues sabemos por la historia de nuestra disciplina (Pavlov, 1970,1972 ) que un simple sonido puede provocar cambios fisiológicos y de comportamiento. Estos resultados se han visto confirmados en deportistas al presentarles palabras e imágenes positivas para modificar estados de activación fisiológica provocados por frustraciones. (Estrada y Pérez Córdoba, 2008: Estrada, Pérez Córdoba y Álvarez, 2010)

Esto, en la práctica real, viene a confirmar que, unas palabras adecuadamente dichas, pueden modificar el nivel emocional -y de activación- o el control atencional, por ejemplo
(Mari, 1994) de los sujetos, de manera que, nuestra intervención, puede pasar por hacer que esas palabras (a modo de autoinstrucciones o de instrucciones de otro, por ejemplo el entrenador) aparezcan -o no aparezcan, si es el caso- haciendo modificar el comportamiento del deportista.

Una vez diseńado el producto, hay que contrastar la adecuación y eficacia del "prototipo" de servicio o producto desarrollado antes de utilizarlo en la intervención. Esto nos lleva a pensar que, la evaluación propuesta en el paso anterior, debe ser continua, pues hemos de conocer los efectos que dicha intervención provoca realmente.

Siguiendo con el símil médico, no bastaría con prescribir el fármaco, sino que habría que comprobar que provoca los efectos oportunos, pues, aunque sabemos que, en general, los produce, podría ocurrir que, en ese organismo -y con esas circunstancias- provocara los efectos contrarios. Ya sabemos que en nuestra Seguridad Social los tiempos de los que se disponen no son lo adecuados, y, si el paciente no va mejorando "ya volverá a la consulta”, o, simplemente, ya lo ingresaremos en un hospital donde podremos hacer esta evaluación mas continua.

En lo psicológico puede ocurrir algo similar, pues, como sabemos por la experimentación con animales, la comida "suele ser" un reforzador útil, pero, ojo, solo en los casos en que hay una privación previa de alimentos - peso ad libitum-, pues, caso contrario, la comida puede funcionar incluso como estímulo aversivo. En este sentido, unas frases muy exigentes a un deportista pueden resultar eficientes en unos casos $-\mathrm{y}$ a unos deportistas- mientras que puede resultar incluso perjudicial en otros momentos $-\mathrm{o}$ con otras personas.

Esto nos debe llevar a la adaptación de los productos o servicios que el profesional tiene disponibles, o al desarrollo de nuevos productos, dado que la simple utilización no resultaría adecuada, eficaz y/o eficiente.

En este sentido, hay que adaptar el diseño a las circunstancias, y, esto, solo es posible de realizar por el profesional que diseña y realiza la intervención -en base a la evaluación propuesta en el apartado anterior- pues, como hemos dicho en otro lugar (Pérez Córdoba, 1997), el teórico tiene como función la de establecer teorías "generales", abstrayendo -y por tanto, generalizando- de los múltiples casos individuales de intervenciones reales o simuladas en el laboratorio, corres- 
pondiendo al aplicado "particularizar" dicha teoría para el caso y el momento - persona y circunstancias- para las que va a proponer dicho diseño de intervención. Tal vez lo ideal sea que haya mas unión entre teoría y aplicación, como plantean Weinberg y Gould, (1996)

Esta adaptación a las circunstancias persigue optimizar la intervención, y no solo proponer una intervención "general" que nos ofrezca altas probabilidades de éxito, "en general", pero que, podría ser, en este caso "en particular", no tan eficaz por no adaptarse a la persona y las circunstancias concretas.

Así, y ahora con el símil del educador, cabría decir que, pese a que "cada maestrillo tiene su librillo" o forma general de impartir su docencia, el psicólogo en la educación ha de intentar "poseer muchos librillos" para poder así adaptar su método a cada sujeto/situación.

Este "tener en cuenta la situación específica" debe tener en cuenta, también, que los cambios se producen de manera gradual -pese a que podamos advertirlos de golpe-de manera que, en el diseńo, no solo se tendrá en cuenta los cambios que se van a introducir, a modo de aparición/desaparición de estímulos -frases, imágenes, etc.-, sino su progresividad, partiendo, siempre, de la línea base del sujeto -detectada en la evaluación previa-. Esto permitirá el cambio gradual en la forma de comportamiento, siendo tan complejo el decidir los elementos a introducir/eliminar como la graduación del cambio que se va a requerir.

Retomando el caso del profesional médico, éste no solo debe decidir qué fármaco va a tomar el paciente -o que cosas va a dejar de comer- sino que debe prescribir en qué cantidades debe hacerlo, teniendo en cuenta que, incluso un simple resfriado - permítasenos la expresión coloquial- necesita de "una semana" para su curación.

¿Se imagina el lector tomarse todo el bote de pastillas completo - por ejemplo de antibióticos- en el primer día para acelerar el proceso?

Es evidente que esto no aceleraría el proceso, mas bien, produciría unos cambios contraproducentes, pues no olvidemos que, todos los fármacos son "veneno", en según qué dosis. En este mismo sentido, unas frases de aprobación, introducidas en el momento oportuno por el entrenador y/o padres, si se producen en exceso, pueden ser tan contraproducentes como el exceso de fármaco ¿podríamos decir que el exceso de información también actúa como el veneno? Lo que sí es cierto que el exceso de reforzadores actúa como "elemento que sacia”, y, por tanto, provoca el efecto contrario en el proceso de aprender.

No creemos que sea necesario abundar en este aspecto, pues la técnica de establecimiento de objetivos ya nos habla suficientemente de ello, tan solo queremos mencionar que, si difícil resulta decidir sobre qué elementos vamos a trabajar, también tiene su dificultad el establecer los criterios de reforzamiento adecuados para que el proceso de cambio no sea mas lento o rápido de lo necesario.

Como diría el dicho popular, hay que estar en el sitio exacto, y en el momento oportuno, sabiendo, además, qué hacer -o dejar de hacer- en cada situación, entendiendo que el "pleno acierto" en "todas" las condiciones es algo menos que imposible -o escasamente probable-, lo que no nos debe llevar a "no hacer nada", sino, más bien, a entender que, lo importante, es ir introduciendo los cambios y comprobando que se van produciendo esos cambios en el comportamiento que queremos modificar.

En este diseño también debemos prever cómo o quién va a introducir los cambios, pues, si bien es cierto que en el laboratorio la aparición/desaparición de estímulos se produce de una forma mecánica y pre-programada de una forma mecánica -en el ordenador-, los "dispensadores de reforzadores" comederos en el laboratorio, padres y entrenadores, por ejemplo, aquí- no son tan fácilmente "manipulables" como lo son en el laboratorio, debiéndose prever, en el diseño, la forma en que dichos dispensadores de estímulos actuarán en la realidad, y debemos prever también si disponemos de recursos suficientes -como profesionales- para provocar esos cambios en los dispensadores de estímulos en la realidad.

En cualquier caso, solo tras un buen diseño, en el que hemos de tener en cuenta todo lo anterior - en la mejor medida posible-, y tras un periodo de prueba - a modo de estudio piloto- se podrá pasar a la puesta en práctica de dicho diseño, aunque esto ya es el paso siguiente.

Cuadro 3. Definición de la intervención.

Continuando con nuestro ejemplo del futbolista, una vez definido el problema (no marca tantos goles como antes en los saques de esquina), y evaluado (su comportamiento incluye autoinstrucciones y pensamientos no adecuados justo en el momento de la ejecución), hemos de pasar al diseño de la intervención.

Así, nuestra intervención, lejos de ser "abstracta" o "poco definida" puede pasar a ser diseñada de una forma mas operativa, pues, el objetivo final está claro: que el deportista deje de emitir dichos comportamientos (pensamientos, etc.) en ese momento y pase a darse "autoinstrrucciones" específicas en dicho momento, al menos en una primera fase de la intervención, pues el objetivo final es que lo automatice y, ni siquiera tenga que darse esa "autoinstrucción”. 
Claro que el problema está ahora en cómo lo vamos a conseguir, y, para ello, lo primero que tenemos que pensar es qué vamos a hacer con los estímulos adecuados para que aparezcan, o para que los hagan aparecer otras personas- y qué o cómo vamos a actuar para que, una vez que aparezca el comportamiento adecuado -y desaparezca el inadecuado- se "refuerce" dicho comportamiento, es decir, se produzca con una mayor tasa.

Teniendo en cuenta que, en este artículo trabajamos con "supuestos", plantearíamos un diseño basado en etapas -la realidad podría hacernos cambiar este diseño y tener que adaptarlo a la persona y las circunstancias reales-, de manera que, en una primera fase algún estímulo externo (por ejemplo una voz del entrenador) puede hacer que el sujeto empiece a decirse las autoinstrucciones en voz alta. Ello le impediría "pensar" en otras cosas no útiles para la eficacia del comportamiento.

En posteriores fases se iría disminuyendo la intensidad de la voz del entrenador (hasta el punto de hacerla desaparecer) y se le pediría al deportista que no se dijera las instrucciones en voz alta sino que las "pensara".

Una última fase nos llevaría q automatizarlo todo de manera que, durante el comportamiento centrara su atención en aquellos elementos de la tarea que le harían ser eficaz (según nos definió el entrenador)

Tendremos que pensar, además, cómo "reforzar" los diferentes avances, y cómo se registrará la evaluación, tendiendo en cuenta que, quien dispensa los reforzadores puede ser el compañero y/o el entrenador -además del autorrefuerzo o el refuerzo generado por la consecución del gol en el entrenamiento.

Todo este "diseño" de la intervención, debe preceder a su puesta en práctica, pues no podemos -o no debemos- actuar "sobre la marcha”, como podría hacerlo el que no es profesional de la psicología -psicólogo de calle, le hemos llamado-, sin que ello nos lleve a ser tan inflexibles que no podamos modificar el diseño en función de la evaluación de los resultados que se van obteniendo (como podría hacerlo un médico al cambiar el tratamiento si se ve necesario)

\section{Puesta en práctica de la intervención diseńada}

Tras los pasos anteriores (definición operativa, evaluación y diseño) nos toca poner en práctica la intervención planificada.

En este punto conviene señalar que existen unas técnicas, que aprendemos a aplicar en situaciones estándar -situaciones teóricas y por tanto aplicaciones estándar- y que se estudian durante la carrera, pero que existen unas condiciones reales -que son las que acabamos de evaluar- en las que hemos de aplicarlas.

Estas condiciones, además, pueden ser cambiantes, tanto los estímulos situacionales externos -o del contexto- como los del propio organismo (Kantor, 1967; Ribes y López, 1985), siendo evidente que dichas condiciones "condicionan" -como su nombre indica- la relación y se disponen de una forma específica. Sí, además, tenemos en cuenta que las relaciones entre ese individuo y ese contexto poseen una historia previa que las pre-dispone (Pérez Córdoba y Llanes, 2010), podremos fácilmente comprender que no hay dos situaciones "exactamente" iguales.

Es decir, que pese a que en la Universidad se nos enseñan las técnicas de intervención psicológicas, en su implementación genérica o teórica -técnica- hemos de actuar con "tacto" para adecuarlas a las circunstancias -en argot deportivo, con cierta táctica. (Pérez Córdoba, 2012b)

Así, y pese a que el diseño lo hemos elaborado ya -en el paso anterior-, el profesional ha de ser competente en la planificación de la intervención específica que va a realizar, previendo, entre otros aspectos, las dificultades que pueden surgir y los aspectos que pudieran poner en peligro o cuestión su eficacia, o incluso los efectos colaterales -no pretendidos- que pudieran resultar negativos, teniendo en cuenta los diferentes escenarios posibles.

Si seguimos con el símil médico, tendremos que tener en cuenta si ese paciente tiene diabetes o no para que no interactúe negativamente con el tratamiento encomendado, pues sucede que dicha medicación no está recomendada para este tipo de pacientes. Del mismo modo tendrá que ver si el contexto -padres, familiares, amigos, etc.- son los adecuados para administrar el tratamiento, si el paciente no está capacitado para ello - por su enfermedad, su edad, etc.-, pues la eficacia del mismo depende, en gran medida, de su correcta administración.

En este sentido, hemos de recordar aquí que, si bien el médico es el que diseña la intervención, no es el que la administra, contando, para ello, con los familiares o el mismo paciente, o, en su caso, con los enfermeros que visitan al paciente o en el propio hospital o centro de salud.

Esto, desgraciadamente no ocurre en psicología, pues, aún, no disponemos de enfermeros para la aplicación -incluso me atrevería a decir que al psicólogo no se le prepara para ello, sino, más bien, para diseñar la intervención, como al médico, aunque esto sería otra reflexión- siendo el propio psicólogo el que debe implementar dicha intervención.

Si esto es, y es el propio psicólogo el que administra la intervención, se le denomina intervención directa, aunque también puede ocurrir que el psicólogo pida a entrenadores, familiares, etc., que sean ellos los que aporten las informaciones - a modo de pastillas- o que eliminen los estímulos aversivos - a modo de quejas de esto, por ejemplo- o, en definitiva, que pongan en práctica dicho diseño. En este caso estaríamos 
hablando de intervención indirecta, muy usada en psicología del deporte.

Sea quien sea quien aporte la estimulación -o la elimine, en su caso-, estas modificaciones pueden consistir tanto en cambios en el contexto, como en cambios en la persona -en las relaciones que éste describe sobre la realidad, sus expectativas, creencias, etc.

Visto así, y dado que el psicólogo trabaja con relaciones, tan efectivo puede ser trabajar con un elemento -el contexto- como con el otro -la persona- de manera que, al cambiar alguno de ellos -o ambos si podemos y si es el caso- pueda modificarse la relación.

Y estos cambios pueden ser propiciados tanto por el propio psicólogo de una forma directa -hablando con el deportista, presentando estímulos asociados con algo, etc.- como indi- recta - haciendo que esto lo haga otra persona. Este último caso es mas parecido al quehacer del médico en una consulta, si bien es cierto que el trabajo del psicólogo, en muchas ocasiones, es más de campo que de despacho, no olvidemos el famoso artículo de Martens sobre batas y chándales (Martens, 1979).

Este último modo, el directo, hace que nuestro trabajo se asemeje más al de educador que al del profesional de la salud.

Por último, ni que decir tiene que el objetivo de la intervención puede ser tanto rehabilitador como preventivo, de manera que, en ambos casos, se favorezcan las relaciones ajustadas al objetivo -ya sea rendimiento, ocio y recreación o bienestar- consiguiéndose, lejos de perfecciones, mejoras en cuanto al grado de ajuste a la consecución de dicho objetivo.

\section{Cuadro 4. Puesta en práctica.}

En el caso del futbolista que nos ocupa, hemos decidido hacer una intervención mixta, de un lado indirecta, con la ayuda de entrenador y compañeros, y de otra directa, para trabajar las creencias y evaluar la evolución en cuanto a su nivel de concentración, focalización de la tarea y control emocional.

En cuanto a la indirecta, contamos con la ayuda de, al menos, dos compañeros y el entrenador. Uno de ellos es el que lanza los saques de esquina, quien, además de señalar hacia donde va a dirigir su lanzamiento -estímulo discriminativo- se señala la cabeza mirando hacia nuestro delantero, lo que hemos convertido, previamente, en señal de que el delantero debe de "pensar" o, mejor dicho, darse las autoinstrucciones pertinentes.

El segundo compañero se sitúa junto al delantero y le indica en voz alta que debe centrar su atención mediante una señal de voz "céntrate". Y, el entrenador es el encargado de animar si se hace bien y de extinguir cuando no se hace adecuadamente, acompańando dicho comportamiento de un recordatorio de cómo deben de realizarse las autoinstrucciones.

Poco a poco haremos que vayan desvaneciéndose dichas estimulaciones hasta que consigamos que se produzca el comportamiento sin ningún estímulo externo.

También contamos con nuestra propia intervención, pues trabajamos a diario con el deportista, contabilizando las ocasiones "correctas" y trabajando estrategias para mejorar sus recursos y habilidades psicológicas para centrar su atención. Además, se ha trabajado el hecho de que, cualquier cambio, sobre todo al principio, suele empeorar la ejecución, siendo su mejoría lenta y dependiente, entre otras cosas, de la cantidad de práctica y de la calidad de la misma.

En este caso, y solo a modo de ejemplo -recordemos que es un supuesto, no un caso real con sus circunstancias específicashemos detectado que el entrenador no suele "reforzar" siempre, por lo que trabajaremos con él mas específicamente de cara a conseguir que sus elogios se produzcan en un mayor número de ocasiones -en cada caso las realidades superan esta ficciones y el psicólogo deberá estar "atento" a ellas para las necesarias adaptaciones.

No olvidemos, por último, que hemos de ajustar los "dispensadores de reforzadores positivos" (entrenadores, por ejemplo) para que refuercen de manera contingente con la respuesta correcta (decir, en voz alta al principio, dónde está el portero, dónde los contrarios y describiendo hacia dónde irá la pelota según los gestos del lanzador, en definitiva, "manipulando" los estimuladores pero no del laboratorio, sino de la realidad).

\section{Evaluación de programas e intervenciones}

Un penúltimo paso consistiría en la evaluación de la intervención, evaluación que debe ser diseñada junto con el diseño de la intervención.

Esto viene a decir que, desde el principio, ya definido el objetivo y diseñada la intervención, debemos planificar el cómo vamos a valorar el cambio producido por nuestra ac- tuación, y si éste va a ser evaluado solo mediante "la opinión subjetiva del cliente" o, mejor, si va a ser medida de alguna otra forma, como test, registro de frecuencias, etc. Es decir, hay que definir qué indicadores se van a utilizar en la medida de la eficacia del tratamiento.

Esta evaluación nos servirá para poder realizar un informe más correcto, en el siguiente y último paso, o para poder analizar si los cambios que se van produciendo, si los hubiese, 
se han debido a nuestras intervenciones, y nos servirá parea poder ir modificando los plazos de nuestras actuaciones o las propias medidas que vamos adoptando.

En definitiva, sería una evaluación continua, que nos serviría para "dar cuenta y razón" de las actuaciones y de los efectos que han producido. También es importante conocer si se han producido efectos colaterales positivos o negativos y otros aspectos relacionados con la eficacia y eficiencia del proceso.

En el símil médico, éste nos debe prescribir continuas analíticas -en la justa medida, sin excesos tampoco- para comprobar la evolución del enfermo. Esta evaluación, no suele producirse en las consultas extra hospitalarias, donde a menudo se tratan las enfermedades leves, pero sí que es común en los hospitales, donde el médico realiza una visita diaria y se realizan continuas mediciones de los indicadores relevantes.

En el caso de la psicología del deporte resulta conveniente la evaluación continua -sin exceso también- de los cambios producidos, y de la correcta dispensación de los estímulos, pues no debemos olvidar que el objetivo de nuestra intervención no es solo conseguir el objetivo establecido, sino el hacerlo en el menor plazo posible y con el menor gasto de recursos posibles, cuestión esta -la de los plazos- que se muestra de mayor importancia en el deporte de alta competición.

La evaluación de programas e intervenciones es un aspecto ya estudiado por otros autores, por lo que no nos extenderemos sobre ello (Hernández Mendo, 2001; Morales, Hernández Mendo y Blanco, 2005)

Cuadro 5. Evaluación de programas e intervenciones.

En el ejemplo que nos ocupa, hemos de medir si se presentan los estímulos tal y como se ha programado, es decir, si el lanzador se toca la cabeza, si el entrenador, previamente, ha dicho en voz alta lo que tenía que decir, si el compañero le grita "concéntrate" y, como dato fundamental, si el sujeto emite (al principio en voz alta y en posteriores ensayos de manera desvanecida hasta no llegar a emitirlas) las autoinstrucciones.

Este registro debe ser lo mas continuo posible, aunque, a medida que se van desvaneciendo estos estímulos, y las autoinstrucciones pasan a ser en voz baja, resulta mas difícil de evaluar. No obstante, tenemos que tener previsto esto y diseñar el modo en que vamos a registrar este hecho, por ejemplo, con un observador -mejor dos- puesto tras la portería y que le cuestionan al delantero -tras la ejecución- si se produjo o no este comportamiento, todo ello, por ejemplo, mediante signos o señas previamente pactadas entre los participantes.

Esta evaluación del registro de la efectividad de la intervención debe llevarse a cabo por indicadores tales como la efectividad de la acción del delantero, en primer lugar -por el porcentaje de goles marcados-, y, la forma en que se va produciendo su comportamiento, en segundo lugar, aunque no debemos olvidar que, muy probablemente, la efectividad tenga que ver con la forma en que se ejecuta (al menos según las indicaciones del técnico en la materia, no olvidemos que nosotros sabemos de cambiar comportamientos, no de ejecuciones técnicas en el deporte)

De esta forma, la evaluación debe servir para que, caso de que no se vayan produciendo los resultados esperados, se puedan ir proponiendo las modificaciones pertinentes -si fuesen necesarias- para conseguir otros resultados, sirviendo además, para que, posteriormente, podamos realizar un adecuado informe final, como seńalaremos en el siguiente punto.

\section{Informe final}

Como último paso, hemos de elaborar un informe de lo sucedido, informe que debe servir para dar a conocer los avances, condiciones, y resultados producidos con la intervención, de manera que se comuniquen esos resultados y se proporcione un feedback adecuado para el sujeto, de manera que, incluso esta información final pueda servir para mantener y/o instaurar posteriores comportamientos.

Este informe debe servir, además, para que no se realicen, por parte del cliente o de sus allegados, falsas interpretaciones sobre las causas del cambio comportamental, pues pudiera ocurrir que, a la postre, se achacaran las causas de la mejoría, a circunstancias un tanto "etéreas" tales como la "suerte", las "meigas" o cualquiera otros factores de difícil localización -si no imposible- pero a la que se suele acudir para explicar los comportamientos y las causas de éstos. En este sentido no debemos olvidar que nosotros mismos queremos seguir trabajando en un futuro, y, así, al conocer los verdaderos motivos del cambio, podrá contar con nosotros en posteriores ocasiones.

Vista la necesidad de evaluar y de presentar informes sobre dichas evaluaciones y cambios de comportamiento, es el momento de plantearnos si dichos informes deben darse al deportista o el entrenador o al club, remitiendo al lector interesado a las normas éticas que figuran en el código deontológico de la profesión (Colegio Oficial de Psicólogos, 2003). Baste ahora con señalar que el informe debe ser confidencial para la persona que nos ocupa como objetivo de la intervención -a menudo el propio deportista-, pudiéndose informar a entrenador y/o club siempre que exista un adecuado consentimiento del deportista en cuestión. Decir, además, que, 
si se trata de menores, esta información, debe ser mucho más cuidadosa en cuanto a su administración.

Un última alusión al símil del médico, nos llevaría a reconocer que, siempre que se produce una intervención médica -no nos referimos, claro está, a la ambulatoria, aunque en ésta, a veces, también se dan estos informes- se entrega un diagnóstico al paciente, informe que, no solo sirve para que éste conozca la evolución de su enfermedad y los fármacos y demás intervenciones que se han producido, sino que, a menudo, incluye también la prescripción de cómo debe comportarse el paciente a partir de entonces y, si fuese necesario, cuándo debe volver a la visita ambulatoria el propio paciente.

En el caso del psicólogo del deporte dicho informe puede y debe tener las mismas funciones, aunque ańadiríamos desde este trabajo - un artículo de una revista científica- el valor de informar a los demás compañeros -y a nosotros mismos en momentos posteriores- de cómo se ha producido la intervención y los resultados que se han ido obteniendo, por ejemplo, mediante un artículo o un capítulo en un libro.

$\mathrm{Ni}$ que decir tiene que este trabajo o informe escrito puede servir también para el teórico, pues, como dijimos antes, la labor de éste consiste en abstraer de muchos casos individuales, por lo que conviene que haya muchos casos individuales, descritos por informes, con los que poder abstraer. De hecho, cada vez existen más revistas que incluyen este tipo de informes sobre intervenciones y no solo los que incluyen grandes estadísticas realizadas sobre un gran número de sujetos que avalen los datos de sus investigaciones.

Este último tipo de informes, los de intervenciones, nos ayudarían a analizar la evolución longitudinal y no tanto la transversal, y, no olvidemos, que la psicología estudia -o debe estudiar- esa evolución individual, entendiendo que no solo la persona es única y diferente -estudio de las diferencias individuales- sino que, además, las circunstancias también son únicas y diferentes - pese a que se puedan establecerse paralelismos entre diferentes situaciones o se puedan manipular las circunstancias para que sean "totalmente iguales" como ocurre en el laboratorio- de manera que, como dijimos en un principio, no existen dos situaciones - que incluyen persona y contexto- iguales, a lo sumo, parecidas o clasificadas en la misma categoría.

\section{Cuadro 6. Informe final.}

En este último apartado sobre el ejemplo, tan solo mencionaremos la necesidad de realizar un informe adecuado sobre nuestra intervención. Informe que debe incluir tanto la evaluación inicial como los diferentes cambios producidos en la conducta del sujeto como los pertinentes cambios propuestos por el psicólogo.

No nos extenderemos en esta parte pero sí que diremos que no se trata de un informe experimental, al uso y según las normas APA, pero sí de un informe en el que se especifiquen los aspectos tratados en este escrito: una definición operativa del problema, los resultados de la evaluación y el esquema de la intervención realizada, así como los resultados de la misma.

$\mathrm{Ni}$ que decir tiene que este informe debe cumplir unos mínimos para que el cliente pueda obtener la información adecuada y pueda seguir las instrucciones pertinentes (si las hubiere), para continuar con la intervención si esto fuese necesario, de cara a que el comportamiento instaurado se mantenga en el tiempo, incluyendo al final unas conclusiones y, si es preciso, unas instrucciones a modo de recomendaciones futuras.

\section{Referencias}

1. Colegio Oficial de Psicología. (2003). Ética y deontología para psicólogos. Madrid: Autor.

2. Estrada, O. y Pérez Córdoba, E. (2008). Palabras e imágenes positivas en la respuesta de ansiedad en deportistas de competición. Cuadernos de Psicología del Deporte, 8(1), 31-45.

3. Estrada, O; Pérez Córdoba, E.A. y Álvarez, M. A. (2010). Presentación de estímulos positivos como procedimiento para controlar la ansiedad en situaciones estresantes. Apuntes de Psicología, 28(2), 125-236.

4. Hernández Mendo, A. (2001). Un cuestionario para evaluar la calidad en programas de actividad física. Revista de Psicología del deporte, 10, 179-196.

1. 5. Infocop online. (2008). Las competencias, elemento fundamental en los nuevos planes de estudios según el proyecto Europsy. Infocop online 30/10/2008. Recuperado de http://www.infocop.es/view_article. asp?id=2084

6. Kantor, J. R. (1967). Psicología interconductual. México: Trillas.
7. Mari, J. (1994). Mejora de la concentración en pista: experiencia en hockey hierba alto rendimiento. ApunIs: Educación física y Deportes, 35, $32-$ 37

8. Martens, R. (1979). About smocks and jocks. Journal of Sport Psychology, 1,94-99.

9. Morales, V.; Hernández Mendo, A. y Blanco, A. (2005). Evaluación de la calidad en los programas de actividad física. Psicothema, 17( 2), 311-317.

10. Pavlov, I. P. (1970). Fisiología y psicología. Madrid: Alianza Editorial.

11. Pavlov, I. P. (1972). Reflejos condicionados e inhibiciones. Barcelona: Ediciones Península.

12. Pérez Córdoba, E. A. (1997). El comportamiento y su análisis en el deporte: de la práctica a la teoría (y viceversa). Revista de Psicología del Deporte, 11, 115-125.

13. Pérez Córdoba, E. A. (2010). Competencias profesionales del psicólogo del deporte. Apuntes de Psicología, 28( 2), 181-194.

14. Pérez Córdoba, E.A. (2011). Fisiología y psicología en el deporte: aspec- 
tos conceptuales y metodológicos. Cuadernos de Psicología del Deporte, 11(2), 81-88.

15. Pérez Córdoba, E.A. (2012a). En qué deben ser competentes los psicólogos del deporte. En H. Gundlach; E. A. Pérez Córdoba; M. Sinatra y G. Tanucci (Eds.), L'arte del movimento. (pp. 199-220). Lecce: Pensa Multimedia s.r.l.

16. Pérez Córdoba, E.A. (2012b): Competencias profesionales del psicólogo del deporte. Conferencia impartida en el VI Congreso Internacional de Medicina y Ciencias Aplicadas al Deporte y a la Actividad Física. La Habana, 30 de Mayo- 1 de Junio de 2012.

17. Pérez Córdoba, E. A. y Caracuel, J. C. (1995). Lenguaje psicológico y comportamiento deportivo: una visión interconductual. En E. Cantón (Ed.), V
Congreso Nacional de Psicología del Deporte (pp. 286-294). Valencia: Universidad de Valencia.

18. Pérez Córdoba, E. A. y Llanes, R. (2010). Aplicaciones de los conocimientos psicológicos en la iniciación deportiva. Papeles del psicólogo, 31(3), 269-280.

19. Ribes, E. y López, F. (1985). Teoria de la conducta: Un análisis de campo y paramétrico. México: Trillas.

20. Riera, J. (1985). Introducción a la psicología del deporte. Barcelona: Fontanella.

21. Roe, R. A. (2002). What makes a competent psychologist? European Psychologist, 7, 192-202. Weinberg, R. S. y Gould, D. (1996). Fundamentos de psicología del deporte y el ejercicio. Barcelona: Ariel. 